\title{
TOPOLOGY FIELDS, TOPOLOGICAL FLOWS AND TOPOLOGICAL ORGANISMS
}

\author{
JAE-RYONG KIM*
}

\begin{abstract}
Topology may described a pattern of existence of elements of a given set $X$. The family $\tau(X)$ of all topologies given on a set $X$ form a complete lattice. We will give some topologies on this lattice $\tau(X)$ using a topology on $X$ and regard $\tau(X)$ a topological space.

A topology $\tau$ on $X$ can be regarded a map from $X$ to $\tau(X)$ naturally. Such a map will be called topology field. Similarly we can also define pe-topology field. If $X$ is a topological flow group with acting group $T$, then naturally we can get a another topological flow $\tau(X)$ with same acting group $T$. If the topological flow $X$ is minimal, we can prove $\tau(X)$ is also minimal.

The disjoint unions of the topological spaces can describe some topological systems (topological organisms). Here we will give a definition of topological organism. Our purpose of this study is to describe some properties concerning patterns of relationship between topology fields and topological organisms.
\end{abstract}

\section{Introduction}

Let $X$ be a topological space with topology $\tau$ and $p$ be a point of $X$. The set of all nbds of $p$ will be denoted by $N(p)$. We can easily verify that $N(p) \cup\{\emptyset\}$ become a topology on $X$ for any point $p$ in $X$. Moreover the collection of all open nbds of $p$ and empty set, that is, $\{V \in \tau \mid p \in V\} \cup\{\emptyset\}$ becomes a topology on $X$ for any point $p \in X$. We will denote such a topology by $\tau_{p}$ and call localized topology of $\tau$ at $p$. Clearly $\tau_{p}$ is weaker than $N(p) \cup\{\emptyset\}$. We will denote the localized topology of the discrete topology $\mathcal{P}(X)$ on $X$ at $p$ by $1_{p}$.

Received August 21, 2012; Accepted November 09, 2012.

2010 Mathematics Subject Classification: Primary 54A10, 57S99; Secondary 93A30.

Key words and phrases: topology (pe topology) field, topological flow, topological organism.

Supported by Kookmin University Research Fund. 
It is easy to show that $(\mathrm{X}, \tau)$ is $T_{0}$ if and only if the localized topologies of $\tau$ at any two points are different with each other. We may regard $X$ as the subset $\left\{1_{p} \mid p \in X\right\}$ of $\tau(X)$ since the localized topologies of the discrete topology at distinct point are different. If we denote $\tau_{p}^{e}$ by $\tau_{p}^{e}=\{U \in \tau \mid p \notin U\} \cup\{X\}$, then it is also a topology on $X$. Moreover $\tau_{p} \cap \tau_{p}^{e}=\{\emptyset, X\}$ and $\tau_{p} \cup \tau_{p}^{e}=\tau$. We will call it point $p$ excluded topology or pe-topology of $\tau$ at $p$.

Theorem 1.1. Let $\tau$ and $\eta$ be two topologies on $X$. Then $\tau=\eta$ if and only if the localized topologies $\tau_{p}, \eta_{p}$ are equal for every point $p$ in X. Moreover $\left(\tau_{p}\right)_{q}=\left(\tau_{q}\right)_{p}$.

This Theorem 1.1 tells us that if some points of the set $X$ change their nbds, that is, change their localized topologies, then the "entire" topology of $X$ will be changed. The family $\tau(X)$ would consist of all topologies on a given fixed set $X$. Here we want to study some conditions concerning the family $\tau(X)$ of all the topologies of the given set $X$.

\section{Topology fields and pe-topology fields on $X$.}

The family $\tau(X)$ of all topologies on $X$ form a complete lattice, that is, given any collection of topologies on $X$, there is a smallest ( respectively largest) topology on $X$ containing( contained in) each member of the collection. Of course, the partial order $\leq$ on $\tau(X)$ is defined by inclusion $\subset$ naturally.

Given a collection of topologies $\left\{\tau_{j} \mid \mathrm{j} \in J\right\} \subset \tau(X)$, we write $\wedge_{j \in J}$ $\tau_{j}$ and $\vee_{j \in J} \tau_{j}$ for their infimum and supremum with respect to this lattice. Of course, $\wedge_{j \in J} \tau_{j}$ is just $\cap_{j \in J} \tau_{j}$, whereas $\vee_{j \in J} \tau_{j}$ has $\cup_{j \in J} \tau_{j}$ as a subbasis. The smallest topology in this lattice $\tau(X)$ is $\{\emptyset, X\}$ and the largest one is $\mathcal{P}(X)$. These topologies will sometimes be denoted by 0 and 1 respectively.

Denote $\tau_{p}(X)=\left\{\eta_{p} \mid \eta \in \tau(X)\right\}$ for a point $p \in X$. Since $\tau(X)$ is a complete lattice, we can easily find that $\tau_{p}(X)$ is a sublattice of $\tau(X)$. The smallest element of this sublattice $\tau_{p}(X)$ is $0_{p}=0$, the largest element is $\mathcal{P}(X)_{p}=1_{p} \neq 1$. We will call this sublattice $\tau_{p}(X)$ as sublattice of all localized topologies at $p$ in $X$. Now we will regard any member $\tau$ of $\tau(X)$ as a map from $X$ to $\cup_{p} \tau_{p}(X) \subset \tau(X)$ defined by $\tau(p)=\tau_{p}$. Hence this map $\tau$ acts like a vector field on $X$. Such a map $f: X \rightarrow \tau(X)$ defined by $f(p) \in \tau_{p}(X)$ will be called topology field on $X[7]$. 
Since $\tau(X)$ is a complete lattice, every topology field is a member of $\tau(X)^{X}$ consisting of all mappings from $X$ to $\tau(X)$. Hence every topology field acts as a $\tau(X)$-fuzzy subset on $X$.

If $f$ is a map from $X$ to $\tau(X)$ defined by $f(p) \in \tau_{p}(X)$, can we find a topology $\zeta$ such that $\zeta_{p}=f(p)$ ? In general, we can not find the correct topology $\zeta$ such that $\zeta_{p}=f(p)$.[7]

Moreover, for every point $p$ of $X$, we can have a map $p r_{p}: \tau(X) \rightarrow$ $\tau_{p}(X)$ defined by $p r_{p}(\tau)=\tau_{p}$. This map $p r_{p}$ also will be called projection from $\tau(X)$ to $\tau_{p}(X)$ [7]. And we can have naturally inclusion map $i_{n}: \tau_{p}(X) \rightarrow \tau(X)$ defined by $i_{n}\left(\zeta_{p}\right)=\zeta_{p} \in \tau(X)$. It is easy to find that the projections $p r_{p}: \tau(X) \rightarrow \tau_{p}(X)$ are monotone.

Similarly denote $\tau_{p}^{e}(X)=\left\{\eta_{p}^{e} \mid \eta \in \tau(X)\right\}$ for a point $p \in X$. Since $\tau(X)$ is a complete lattice, we can easily observe that $\tau_{p}^{e}(X)$ is a sublattice of $\tau(X)$. The smallest element of this sublattice $\tau_{p}^{e}(X)$ is $0_{p}^{e}=0$, but the largest element is $\mathcal{P}(X)_{p}^{e}=1_{p}^{e} \neq 1$. We will call this sublattice $\tau_{p}^{e}(X)$ as sublattice of all pe-localized topologies at $p$ in $X$.

Now we regard any member $\tau$ of $\tau(X)$ as a map from $X$ to $\cup_{p} \tau_{p}^{e}(X)$ $\subset \tau(X)$ defined by $\tau(p)=\tau_{p}^{e}$. Hence this map $\tau$ acts also like a vector field on $X$. In order to avoid the confusion with topology field, we will use symbol $\tau^{e}$ instead of this map $\tau$. Such a map $g$ from $X$ to $\cup_{p} \tau_{p}^{e}(X) \subset \tau(X)$ defined by $g(p) \in \tau_{p}^{e}(X) \subset \tau(X)$ will be called petopology field on $X$. And now we can have a map $p r_{p}^{e}$ from $\tau(X)$ onto $\tau_{p}^{e}(X) \subset \tau(X)$ defined by $p r_{p}^{e}(\tau)=\tau_{p}^{e}$ for every point $p$ of $X$. This map $p r_{p}^{e}$ also will be called pe-projection from $\tau(X)$ to $\tau_{p}^{e}(X)$.

Let $(X, \tau)$ be a topological space. We want to find some topologies on $\tau(X)$ induced by the given topology $\tau$ which provides the continuity of topology fields and projections.

Definition 2.1. [7] Let $(X, \tau)$ be a topological space, and $G \in \tau$. Let $i(G)=\{\zeta \in \tau(X) \mid G \in \zeta\}$ and denote $\epsilon=\{i(G) \mid G \in \tau\}$, a family of subset of $\tau(X)$. Then there is exactly one topology $I n_{\tau}$ on $\tau(X)$ with $\epsilon$ as a subbasis. We will call this topology as inner topology induced by the topology $\tau$. And the relative topology of $I n_{\tau}$ in $\tau_{p}(X)$ will be denoted by $\mathcal{R} I n_{\tau}$ briefly. Note that $I n_{1}$ need not be the discrete topology in $\tau(X)$.

Theorem 2.2. $\left(\tau(X), I n_{1}\right)$ is $T_{0}$ space.

Proof. Let $\eta, \zeta \in \tau(X)$ with $\eta \neq \zeta$. There there exists at least one $G \in 1$ which is included in the one of them and is excluded from the other one. Say, $G \in \eta$ and $G \notin \zeta$. Hence $\eta \in i(G)$ and $\zeta \notin i(G)$. 
If $\zeta \leq \eta$, then $\forall G \in \zeta, G \in \eta$. That is, if $i(G) \ni \zeta$, then $i(G) \cap\{\eta\} \neq$ $\emptyset$. This implies $\zeta \in \overline{\{\eta\}}$. Conversely $\zeta \in \overline{\{\eta\}}$ implies $\zeta \leq \eta$. If this relation holds we say that $\zeta$ is a specialization of $\eta[9]$. For any $\eta \in \tau(X)$ we will denote the subset $\{\zeta \in \tau(X) \mid \zeta \geq \eta\}$ by $\uparrow(\eta)$. (We shall also use later the notation $\downarrow(\eta)$ for $\{\zeta \in \tau(X) \mid \zeta \leq \eta\}$. Then since $i(G)=\{\zeta \in$ $\tau(X) \mid G \in \zeta\}, i(G)=\uparrow(\{\emptyset, X, G\})$. Hence $\zeta \in \overline{\{\eta\}}$ iff $\zeta \leq \eta$. Since Alexandrov topology $\Upsilon$ on $\tau(X)$ is the collection of all upper sets in $\tau(X)$ (i.e. sets $U$ such that $\eta \in U$ and $\eta \leq \zeta$ imply $\zeta \in U)$ [9], $i(G) \in \Upsilon$. Hence we have the following result

Theorem 2.3. If $\tau \leq \zeta \leq 1$, then $\operatorname{In}_{\tau} \leq \operatorname{In}_{\zeta} \leq I n_{1} \leq \Upsilon$.

Proof. Let $i(G) \in I n_{\tau}$. Then $G \in \tau \leq \zeta$. Hence $G \in \zeta$. Consequently $i(G)=\{\alpha \in \tau(X) \mid G \in \alpha\} \in I n_{\zeta}$. This implies $I n_{\tau} \leq I n_{\zeta}$. Since every subbasic open set $i(G)$ is a upper set, every open set must be an upper set(cf. proposition 1.8 II [9]). This completes the proof.

Now we consider In as a map from $\tau(X)$ to $\tau(\tau(X))$ defined by $\operatorname{In}(\eta)=I n_{\eta}$. Then we have some results:

Theorem 2.4. In $:(\tau(X), \Upsilon) \rightarrow(\tau(\tau(X)), \Upsilon)$ is continuous.

Proof. Let $\zeta \in \tau(X)$ and $K$ is a neighborhood of $\operatorname{In}(\zeta)=\operatorname{In}_{\zeta}$. Then $K$ is a upper set in $\tau(\tau(X))$. On the other hand the upper set $\uparrow(\zeta)$ in $\tau(X)$ is a nbd of $\zeta$. We will show that $\operatorname{In}(\uparrow(\zeta)) \subset K$. Let $\delta \in \uparrow(\zeta)$. Then $\delta \geq \zeta$ and $I n_{\delta} \geq I n_{\zeta}$. Hence we have $I n_{\delta} \in K$.

Theorem 2.5. $\left(\left(\tau(X), I n_{\tau}\right), \wedge\right)$ and $((\tau(X), \Upsilon), \wedge)$ are topological monoids.

Proof. We will prove only the case of $\left(\left(\tau(X), I n_{\tau}\right), \wedge\right)$. The other case is similar. First closeness of the operation $\wedge$ is trivial. Moreover we can easily get the associative law. The largest element 1 in $\tau(X)$ is the identity element for this operation $\wedge$. Now we will prove that the binary operation $\wedge: \tau(X) \times \tau(X) \rightarrow \tau(X)$ is continuous. Let $i(G)$ be an nbd of $\zeta \wedge \eta$. Then $G \in \zeta$ and $G \in \eta$. Thus $i(G) \times i(G)$ is a nbd of $(\zeta, \eta)$ and $\wedge(i(G) \times i(G)) \subset i(G)$. This completes the theorem.

Theorem 2.6. [7] Topology field $\zeta:(X, \tau) \rightarrow\left(\tau(X), I n_{\tau}\right)$ and projection $\operatorname{pr}_{p}:\left(\tau(X), I n_{\tau}\right) \rightarrow\left(\tau_{p}(X), \mathcal{R} I n_{\tau}\right)$ are continuous. Moreover inclusion $i_{n}:\left(\tau_{p}(X), R I n_{\tau}\right) \rightarrow\left(\tau(X), I n_{\tau}\right)$ is continuous.

Proof. Let $p \in X$ and $i(G)$ be a subbasic open nbd of $\zeta(p)=\zeta_{p}$. Then $G \in \zeta_{p}$. This implies $p \in G \in \zeta$. Moreover since $G \in \tau, G$ is a nbd of $p$. Hence if $q \in G, \zeta(q)=\zeta_{q} \in i(G)$, so that $\zeta(G) \subset i(G)$. This shows that topology 
field $\zeta$ is continuous. Now let $\eta \in \tau(X)$ and $i(G) \cap \tau_{p}(X)$ be a subbasic open nbd of $\operatorname{pr}_{p}(\eta)=\eta_{p}$. Then $G \in \eta_{p}$. Consequently $p \in G \in \eta$. This means that $i(G)$ is a nbd of $\eta$. Moreover $\operatorname{pr}_{p}(i(G)) \subset i(G) \cap \tau_{p}(X)$. In fact, if $\zeta \in i(G)$, then $G \in \zeta$. Since $p \in G, G \in \zeta_{p}$. Hence $\zeta_{p} \in i(G)$. This means that projection $p r_{p}$ is continuous. Finally the continuity of inclusion is natural.

Let $f:(X, \tau) \rightarrow(Y, \eta)$ be a continuous surjective map. If we define a map $f_{*}: \tau(X) \rightarrow \tau(Y)$ by $f_{*}(w)=\left\{U \subset Y \mid f^{-1}(U) \in w\right\}$, then $f_{*}(0)=0$ and $f_{*}(1)=1$. Let $\omega \in \tau(X)$. For any subbasic open neighborhood $i(G)$ of $f_{*}(\omega), G \in f_{*}(\omega)$. Thus $f^{-1}(G) \in \omega$. Hence $\omega \in i\left(f^{-1}(G)\right)$. So that $i\left(f^{-1}(G)\right)$ is an open neighborhood of $\omega$. Conversely, if $\zeta \in$ $i\left(f^{-1}(G)\right)$ then $f^{-1}(G) \in \zeta, G \in f_{*}(\zeta), f_{*}(\zeta) \in i(G)$, and $\zeta \in f_{*}^{-1}(i(G))$. Consequently we have

$$
f_{*}^{-1}(i(G))=i\left(f^{-1}(G)\right) .
$$

Note that $i(G) \cup\{0\}$ is also complete sublattice of $\tau(X)$ for a $G \in \tau$. We will denote this sublattice $i(G) \cup\{0\}$ by $i_{F}(G)$. Then naturally we can restrict domain of definition of this map $f_{*}$ to $i_{F}(H)$ for some open $H$ in $X$. Hence we can have $f_{*}: i_{F}\left(f^{-1}(G)\right) \rightarrow i_{F}(G)$ for each open $G$ in $(Y, \eta)$.

Theorem 2.7. Let $f:(X, \tau) \rightarrow(Y, \eta)$ be a continuous bijective map. Then the induce map $f_{*}: i_{F}\left(f^{-1}(G)\right) \rightarrow i_{F}(G)$ is bijective for each open $G$ in $(Y, \eta)$.

Proof. In fact, since $f: X \rightarrow Y$ is continuous, for each open $G$ in $(Y, \eta), f^{-1}(G) \in \tau$. If $\zeta \in i_{F}\left(f^{-1}(G)\right)$, then $\zeta=0$ or $f^{-1}(G) \in \zeta$. Hence $f_{*}(0)=0 \in i_{F}(G)$ or $G \in f_{*}(\zeta)$. This implies $f_{*}(\zeta) \in i_{F}(G)$. Now let $f_{*}(\alpha)=f_{*}(\beta)$ for $\alpha, \beta \in i_{F}\left(f^{-1}(G)\right)$. Then for all $U \in \alpha, U=$ $f^{-1}(f(U)) \in \alpha$. So that $f(U) \in f_{*}(\alpha)=f_{*}(\beta)$. Hence $U \in \beta$. So that $\alpha=\beta$. This implies that $f_{*}$ is injective. Now let $\delta(\neq 0) \in i_{F}(G)$. Then $\vartheta=\left\{f^{-1}(U) \mid U \in \delta\right\} \in i_{F}\left(f^{-1}(G)\right)$. And $f_{*}(\vartheta)=\delta$. This completes the proof.

Theorem 2.8. Let $f:(X, \tau) \rightarrow(Y, \eta)$ be a continuous map. If we define a map $f_{*}: \tau(X) \rightarrow \tau(Y)$ by $f_{*}(w)=\left\{U \subset Y \mid f^{-1}(U) \in w\right\}$, then the map $f_{*}$ is continuous. If $\gamma \leq \delta$, then $f_{*}(\gamma) \leq f_{*}(\delta)$ and $f_{*}(\tau) \geq \eta$. And for any topology field $\zeta$, the diagram

$$
\begin{array}{crr}
(X, \tau) & \stackrel{f}{\longrightarrow} & (Y, \eta) \\
\downarrow \zeta & & \downarrow f_{*}(\zeta)
\end{array}
$$




$$
\begin{array}{ccc}
\left(\tau(X), I n_{\tau}\right) & \stackrel{f_{*}}{\longrightarrow} & \left(\tau(Y), I n_{\eta}\right) \\
\downarrow p r_{p} & & \downarrow p r_{f(p)} \\
\left(\tau_{p}(X), \mathcal{R} I n_{\tau}\right) & \stackrel{f_{*}}{\longrightarrow} & \left(\tau_{f(p)}(Y), \mathcal{R} I n_{\eta}\right)
\end{array}
$$

commutes. If, furthermore, $(Z, \theta)$ is a topological space and $g:(Y, \eta)$ $\rightarrow(Z, \theta)$ is a map, then

$$
(g \circ f)_{*}=g_{*} \circ f_{*} .
$$

Finally, if $f:(X, \tau) \rightarrow(X, \tau)$ is the identity homeomorphism, then so is $f_{*}$.

Proof. Let $\omega \in \tau(X)$. For any subbasic open neighborhood $i(G)$ of $f_{*}(\omega), G \in f_{*}(\omega)$. Thus $f^{-1}(G) \in \omega$. Hence $\omega \in i\left(f^{-1}(G)\right)$. So that $i\left(f^{-1}(G)\right.$ is a open neighborhood of $\omega$. Conversely if $\zeta \in i\left(f^{-1}(G)\right)$, then $f^{-1}(G) \in \zeta . G \in f_{*}(\zeta) . f_{*}(\zeta) \in i(G) . \zeta \in f_{*}^{-1}(i(G))$. Consequently we have

$$
f_{*}^{-1}(i(G))=i\left(f^{-1}(G)\right) .
$$

This means $f_{*}$ is continuous. Moreover

$$
\begin{aligned}
f_{*}\left(\zeta_{p}\right) & =\left\{U \mid f^{-1}(U) \in \zeta_{p}\right\}=\left\{U \mid p \in f^{-1}(U) \in \zeta\right\} \\
& =\left\{U \mid f(p) \in U, f^{-1}(U) \in \zeta\right\}=\left\{U \mid U \in f_{*}(\zeta), f(p) \in U\right\} \\
& =f_{*}(\zeta)_{f(p)} .
\end{aligned}
$$

This implies upper diagram commutes. And similarly we can have the lower diagram commutes. All other statements follow directly from the definitions.

Consequently for a fixed topology $\zeta$ we can have a continuous map defined by $\wedge_{\zeta}: \tau(X) \rightarrow \tau(X), \wedge_{\zeta}(\eta)=\zeta \wedge \eta$. Let $f:(X, \tau) \rightarrow(Y, \eta)$ be a continuous map. If we define a map $f_{*}: \tau(X) \rightarrow \tau(Y)$ by $f_{*}(w)=\{U \subset$ $\left.Y \mid f^{-1}(U) \in w\right\}$, then

$$
\begin{aligned}
f^{*}(\zeta \wedge \eta)=\left\{U \mid f^{-1}(U) \in \zeta \wedge \eta\right\} & =\left\{U \mid f^{-1}(U) \in \zeta\right\} \wedge\left\{U \mid f^{-1}(U) \in \eta\right\} \\
& =f^{*}(\zeta) \wedge f^{*}(\eta) .
\end{aligned}
$$

Thus we have the following commutative diagram

$$
\begin{array}{ccr}
\left(\tau(X), I n_{\tau}\right) & \stackrel{f_{*}}{\longrightarrow} & \left(\tau(Y), I n_{\eta}\right) \\
\quad \downarrow \wedge_{\zeta} & & \downarrow \wedge_{f^{*}(\zeta)} \\
\left(\tau(X), I n_{\tau}\right) & \stackrel{f_{*}}{\longrightarrow} & \left(\tau(Y), I n_{\eta}\right) .
\end{array}
$$

Using the same method we can also have next theorem 
Corollary 2.9. [7] Let $f:(X, \tau) \rightarrow(Y, \eta)$ be a continuous map. If we define a map $f_{*}^{\eta}: \tau(X) \rightarrow \tau(Y)$ by $f_{*}^{\eta}(w)=\left\{U \in \eta \mid f^{-1}(U) \in w\right\}$, then the map $f_{*}^{\eta}$ is continuous and $f_{*}^{\eta}(\tau)=\eta$. Moreover, for any topology field $\zeta$, the diagram

$$
\begin{array}{ccc}
(X, \tau) & \stackrel{f}{\longrightarrow} & (Y, \eta) \\
\downarrow \zeta & & \downarrow f_{*}^{\eta}(\zeta) \\
\left(\tau(X), I n_{\tau}\right) & \stackrel{f_{*}^{\eta}}{\longrightarrow} & \left(\tau(Y), I n_{\eta}\right) \\
\downarrow p r_{p} & & \downarrow p r_{f(p)} \\
\left(\tau_{p}(X), \mathcal{R} I n_{\tau}\right) & \stackrel{f_{*}^{\eta}}{\longrightarrow} & \left(\tau_{f(p)}(Y), \mathcal{R} I n_{\eta}\right)
\end{array}
$$

commutes. If, furthermore, $(Z, \theta)$ is a topological space and $g:(Y, \eta)$ $\rightarrow(Z, \theta)$ is a map, then

$$
(g \circ f)_{*}^{\theta}=g_{*}^{\theta} \circ f_{*}^{\eta} .
$$

Now we will give another topology on $\tau(X)$ which provide the continuity of pe-topology fields and pe-projections.

Definition 2.10. Let $(X, \tau)$ be a topological space, and $G \in \tau$. Let $o(G)=\{\zeta \in \tau(X) \mid G \notin \zeta\}$ and denote $\epsilon_{o}=\{o(G) \mid G \in \tau\}$, a family of subset of $\tau(X)$ with $o(X)=o(\emptyset)=\emptyset$. Then there is exactly one topology $O u t_{\tau}$ on $\tau(X)$ with $\epsilon_{o}$ as a subbasis. We will call this topology outer topology induced by the topology $\tau$. And the relative topology of $O u t_{\tau}$ in $\tau_{p}(X)$ will be denoted by $\mathcal{R O u t} \tau$ briefly.

Using the notation $\uparrow$ we can describe $o(G)$ by $\tau(X)-i(G)=\tau(X)-\uparrow$ $(\{\emptyset, X, G\})$. Note that $o(G) \cup\{1\}$ is also complete lattice for a $G \in \tau$. Also we will denote this sublattice $o(G) \cup\{1\}$ by $o_{F}(G)$.

Theorem 2.11. $\left(\tau(X)\right.$, Out $\left._{1}\right)$ is $T_{0}$ space.

Proof. Let $\eta, \zeta \in \tau(X)$ with $\eta \neq \zeta$. There there exists at least one $G \in 1$ which is included in one of them and is excluded from the other one. Say, $G \in \eta, G \notin \zeta$. Hence $\eta \notin o(G), \zeta \in o(G)$.

If $\zeta \leq \eta$, then $\forall G \notin \eta, G \notin \zeta$. That is, if $o(G) \ni \eta$, then $o(G) \cap\{\zeta\} \neq \emptyset$. This implies $\eta \in \overline{\{\zeta\}}$. Conversely $\eta \in \overline{\{\zeta\}}$ implies $\zeta \leq \eta$. Hence $\eta \in \overline{\{\zeta\}}$ iff $\zeta \leq \eta$. Since $o(G)=\{\zeta \in \tau(X) \mid G \notin \zeta\}, o(G)=\tau(X)-$ $\uparrow(\{\emptyset, X, G\})$ is a lower set(cf. $2.1 \mathrm{I}[9])$. Now we will define a topology $\perp$ to be the collection of all lower sets in $\tau(X)$. It is clearly a topology, since it is closed under arbitrary unions and intersections. Hence we have 
Theorem 2.12. If $\tau \leq \zeta \leq 1$, then Out $_{\tau} \leq$ Out $_{\zeta} \leq$ Out $_{1} \leq \perp$.

Proof. We will prove the case of $O u t_{\tau} \leq O u t_{\zeta}$. Let $o(G) \in O u t_{\tau}$. Then $G \in \tau$ and consequently $G \in \zeta$. Hence $o(G)=\{\alpha \in \tau(X) \mid G \notin$ $\alpha\} \in$ Out $_{\zeta}$. Since every subbasic open set $o(G)$ is a lower set, every open set must be an lower set. This completes the proof.

Now we consider Out as a map from $\tau(X)$ to $\tau(\tau(X))$ defined by Out $(\eta)=$ Out ${ }_{\eta}$. Then we have

Theorem 2.13. In $:(\tau(X), \perp) \rightarrow(\tau(\tau(X)), \perp)$ is continuous.

Proof. Let $\zeta \in \tau(X)$ and $K$ be a neighborhood of $O u t(\zeta)=$ Out $_{\zeta}$. Then $K$ is a lower set in $\tau(\tau(X))$. On the other hand the lower set $\downarrow(\zeta)$ in $\tau(X)$ is a nbd of $\zeta$. We will show that $O u t(\downarrow(\zeta)) \subset K$. Let $\delta \in \downarrow(\zeta)$. Then $\delta \leq \zeta$ and Out $_{\delta} \leq$ Out $_{\zeta}$. Hence we have Out $\in K$.

Theorem 2.14. $\left(\left(\tau(X)\right.\right.$, Out $\left.\left._{\tau}\right), \vee\right)$ and $((\tau(X), \perp), \vee)$ are topological monoids.

Proof. We will prove only the case of $\left(\left(\tau(X), O u t_{\tau}\right), \vee\right)$. The other case is similar. First closeness of the operation $\vee$ is trivial. Moreover we can easily get the associative law. The smallest element 0 in $\tau(X)$ is the identity element for this operation $\vee$. Now we will prove that the binary operation $\vee: \tau(X) \times \tau(X) \rightarrow \tau(X)$ is continuous. Let $o(G)$ be an nbd of $\zeta \vee \eta$. Then $G \notin \zeta$ and $G \notin \eta$. Thus $o(G) \times o(G)$ is a nbd of $(\zeta, \eta)$ and $\vee(o(G) \times o(G)) \subset o(G)$. This completes the theorem.

Consequently for a fixed topology $\zeta$ we can have a continuous map defined by $\vee_{\zeta}: \tau(X) \rightarrow \tau(X), \vee_{\zeta}(\eta)=\zeta \vee \eta$.

Theorem 2.15. Let $\tau \leq \delta$. Then the pe-topology field $\delta^{e}:(X, \tau) \rightarrow$ $\left(\tau(X)\right.$, Out $\left._{\tau}\right)$ and pe-projection $\operatorname{pr}_{p}^{e}:\left(\tau(X)\right.$, Out $\left._{\tau}\right) \rightarrow\left(\tau_{p}^{e}(X), \mathcal{R O u t}_{\tau}\right)$ are also continuous.

Proof. Let $p \in X$ and $o(G)$ be a subbasic open nbd of $\delta^{e}(p)=\delta_{p}^{e}$. Then $G \notin \delta_{p}^{e}=\{A \in \delta \mid p \notin A\} \cup\{X\}$. This implies $p \in G$. Since $G \in \tau, G$ is a nbd of $p$. If $q \in G, G \notin \delta^{e}(q)=\delta_{q}^{e}$. Hence $\delta_{q}^{e} \in o(G)$. This implies that $\delta^{e}(G) \subset o(G)$. It implies that the pe-topology field $\delta^{e}$ is continuous.

Now let $\eta \in \tau(X)$ and $o(G) \cap \tau_{p}^{e}(X)$ be a subbasic open nbd of $\operatorname{pr}_{p}^{e}(\eta)$ $=\eta_{p}^{e}$. Hence we get $p \in G$. For any $\zeta \in o(G)$ we have $G \notin \zeta$ and $G \notin \zeta_{p}^{e}$. This implies $\zeta_{p}^{e} \in o(G) \cap \tau_{p}^{e}(X)$. Consequently $\operatorname{pr}_{p}^{e}(o(G)) \subset o(G) \cap \tau_{p}^{e}(X)$. This means that pe-projection $p r_{p}^{e}$ is continuous. 
Let $f:(X, \tau) \rightarrow(Y, \eta)$ be a continuous surjective map. If we define a $\operatorname{map} f_{*}: \tau(X) \rightarrow \tau(Y)$ by $f_{*}(w)=\left\{U \subset Y \mid f^{-1}(U) \in w\right\}$, then $f_{*}(0)=0$ and $f_{*}(1)=1$. Similarly as in the case of inner topology we can restrict domain of definition of this map $f_{*}$ to $o_{F}(G)$ for some open $G$ in $X$. We then have $f_{*}: o_{F}\left(f^{-1}(H)\right) \rightarrow o_{F}(H)$ for each open $H$ in $(Y, \eta)$. In fact, since $f: X \rightarrow Y$ is continuous, for each open $H$ in $(Y, \eta), f^{-1}(H) \in \tau$. If $\zeta \in o_{F}\left(f^{-1}(H)\right)$, then $\zeta=1$ or $f^{-1}(H) \notin \zeta$. Hence $f_{*}(1)=1 \in o_{F}(H)$ or $H \notin f_{*}(\zeta)$. This implies $f_{*}(\zeta) \in o_{F}(H)$. Moreover if $f:(X, \tau) \rightarrow(Y, \eta)$ is injective, $f_{*}: o_{F}\left(f^{-1}(H)\right) \rightarrow o_{F}(H)$ is surjective. For $\delta(\neq 1) \in o_{F}(H)$, $\vartheta=\left\{f^{-1}(U) \mid U \in \delta\right\} \in o_{F}\left(f^{-1}(H)\right)$. And $f_{*}(\vartheta)=\delta$. Hence we have

Theorem 2.16. Let $f:(X, \tau) \rightarrow(Y, \eta)$ be a continuous bijective map. Then the induce map $f_{*}: i_{F}\left(f^{-1}(H)\right) \rightarrow i_{F}(H)$ is bijective for each open $H$ in $(Y, \eta)$.

Theorem 2.17. Let $\tau \leq \zeta$. Let $f:(X, \tau) \rightarrow(Y, \eta)$ be a continuous map. If we define a map $f_{*}: \tau(X) \rightarrow \tau(Y)$ by $f_{*}(w)=\left\{U \subset Y \mid f^{-1}(U) \in\right.$ $w\}$, then the map $f_{*}$ is continuous and $f_{*}(\tau) \geq \eta$. Moreover, for any topology field $\zeta$, the diagram

$$
\begin{array}{ccc}
(X, \tau) & \stackrel{f}{\longrightarrow} & (Y, \eta) \\
\downarrow \zeta_{p}^{e} & & \downarrow f_{*}(\zeta)_{f(p)}^{e} \\
\left(\tau(X), \text { Out }_{\tau}\right) & \stackrel{f_{*}}{\longrightarrow} & \left(\tau(Y), \text { Out }_{\eta}\right) \\
\downarrow p r_{p}^{e} & & \downarrow p r_{f(p)}^{e} \\
\left(\tau_{p}(X), \text { ROut }_{\tau}\right) & \stackrel{f_{*}}{\longrightarrow} & \left(\tau_{f(p)}(Y), \text { ROut }_{\eta}\right)
\end{array}
$$

commutes. If, furthermore, $(Z, \theta)$ is a topological space and $g:(Y, \eta)$ $\rightarrow(Z, \theta)$ is a map, then

$$
(g \circ f)_{*}=g_{*} \circ f_{*} .
$$

Finally, if $f:(X, \tau) \rightarrow(X, \tau)$ is the identity homeomorphism, then so is $f_{*}$.

Proof. Let $\omega \in \tau(X)$. For any subbasic open neighborhood $o(G)$ of $f_{*}(\omega), G \notin f_{*}(\omega)$. Thus $f^{-1}(G) \notin \omega$. Hence $\omega \in o\left(f^{-1}(G)\right)$. So that $o\left(f^{-1}(G)\right.$ is a open neighborhood of $\omega$. Conversely if $\zeta \in o\left(f^{-1}(G)\right)$, then $f^{-1}(G) \notin \zeta . G \notin f_{*}(\zeta) . f_{*}(\zeta) \in o(G) . \zeta \in f_{*}^{-1}(o(G))$. Consequently we have

$$
f_{*}^{-1}(o(G))=o\left(f^{-1}(G)\right) .
$$

This means $f_{*}$ is continuous. And we can easily see that the diagram commutes. Moreover the other statements follow directly from the definitions. 
Using the same method in above theorem we can have

Corollary 2.18. Let $\tau \leq \zeta$. Let $f:(X, \tau) \rightarrow(Y, \eta)$ be a continuous map. If we define a map $f_{*}^{\eta}: \tau(X) \rightarrow \tau(Y)$ by $f_{*}^{\eta}(w)=\left\{U \in \eta \mid f^{-1}(U) \in\right.$ $w\}$, then the map $f_{*}^{\eta}$ is continuous and $f_{*}^{\eta}(\tau)=\eta$. Moreover, for any topology field $\zeta$, the diagram

$$
\left.\begin{array}{ccc}
(X, \tau) & \stackrel{f}{\longrightarrow} & (Y, \eta) \\
\downarrow \zeta_{p}^{e} & \downarrow f_{*}^{\eta}(\zeta)_{f(p)}^{e} \\
\left(\tau(X), \text { Out }_{\tau}\right) & \stackrel{f_{*}^{\eta}}{\longrightarrow} & \left(\tau(Y), \text { Out } t_{\eta}\right) \\
\downarrow p r_{p}^{e} & & \downarrow p r_{f(p)}^{e} \\
\left(\tau_{p}(X), \text { ROut }_{\tau}\right) & \stackrel{f_{*}^{\eta}}{\longrightarrow} & \left(\tau_{f(p)}(Y),\right. \text { ROut }
\end{array}\right)
$$

commutes. If, furthermore, $(Z, \theta)$ is a topological space and $g:(Y, \eta)$ $\rightarrow(Z, \theta)$ is a map, then

$$
(g \circ f)_{*}^{\theta}=g_{*}^{\theta} \circ f_{*}^{\eta} .
$$

Finally, if $f:(X, \tau) \rightarrow(X, \tau)$ is the identity homeomorphism, then so is $f_{*}$.

Let $f:(X, \tau) \rightarrow(Y, \eta)$ be a continuous map. Then we have

$$
\begin{aligned}
f^{*}(\zeta \vee \eta)=\left\{U \mid f^{-1}(U) \in \zeta \vee \eta\right\} & \geq\left\{U \mid f^{-1}(U) \in \zeta\right\} \vee\left\{U \mid f^{-1}(U) \in \eta\right\} \\
& =f^{*}(\zeta) \vee f^{*}(\eta)
\end{aligned}
$$

Thus if $\tau \leq \zeta$, then we have the following commutative diagram

$$
\begin{array}{crr}
\left(\tau(X), \text { Out }_{\tau}\right) & \stackrel{f_{*}}{\longrightarrow} & \left(\tau(Y), \text { Out }_{\eta}\right) \\
\downarrow \vee_{\zeta} & & \downarrow \vee_{f^{*}(\zeta)} \\
\left(\tau(X), \text { Out }_{\tau}\right) & \stackrel{f_{*}}{\longrightarrow} & \left.(\tau)(Y), \text { Out }_{\eta}\right) .
\end{array}
$$




\section{Topological flow $(X, T, \pi)$ and topologies on $X$}

A topological flow(or transformation group) is a triple $(X, T, \pi)$, where $X$ is a topological space(phase space), $T$ is a topological group(acting group), and $\pi$ is a continuous map from $X \times T$ to $X$ satisfying

(i) $\pi(x, e)=x \quad(x \in X, e$ the identity of $T)$

(ii) $\pi(\pi(x, s), t)=\pi(x, s t) \quad(x \in X, s, t \in T)$.

Each $t \in T$ defines a continuous map $\pi^{t}$ from $X$ to $X$ by $\pi^{t}(x)=\pi(x, t)$. If $t, s \in T$, then it is immediate that $\pi^{s} \pi^{t}=\pi^{t s}$; in particular, $\pi^{t} \pi^{t^{-1}}=\pi^{e}$, the identity map of $X$, so each $\pi^{t}$ is a homeomorphism of $X$ onto itself, with $\left(\pi^{t}\right)^{-1}=\pi^{t^{-1}}$. In general, we will identify an element $t \in T$ with the homeomorphism $\pi^{t}$ of $X$. Thus $T$ may be regard as a subgroup of the total homeomorphism group of $X$. Moreover, the topology of acting group is really not important. For this reason we will frequently assume that the acting group $T$ has the discrete topology.

TheOREM 3.1. If $(X, T, \pi)$ is a flow with discrete acting group $T$, we have a flow $\left(\tau(X), T, \pi_{*}\right)$ with the same acting group $T$.

Proof. Here we will prove the theorem only in the case of $\tau(X)$ which has outer topology $I n_{\tau}$ induced by $\tau$. The other case is due to the same method. Since each $t \in T$ defines a continuous map(homeomorphism) $\pi^{t}(=t)$ of $X$ to $X$ by $\pi^{t}(x)(=t(x))=\pi(x, t)=x t$, we can get induced continuous map(homeomorphism) $t_{*}$ of $\tau(X)$ to itself by theorem 2.8. Thus we can define a map $\pi_{*}$

$\pi_{*}: \tau(X) \times T \longrightarrow \tau(X), \quad \pi_{*}(\zeta, t)=\zeta t=t_{*}(\zeta)=\left\{U \subset X \mid t^{-1}(U) \in \zeta\right\}$.

Then this map $\pi_{*}$ is well defined and continuous. Indeed, for a open subset $G$ of $X, t^{-1}(G)$ is also open. Moreover, by theorem 2.8, we can get $\pi_{*}\left(i\left(t^{-1}(G)\right), t\right) \subset i(G)$. This implies that the map $\pi_{*}$ is continuous. Finally we can easily find that $\pi_{*}(\zeta, e)=\zeta$ and $\pi_{*}\left(\pi_{*}(\zeta, s), t\right)=\pi_{*}(\zeta, s t)$.

Theorem 3.2. [7] Let $(X, T, \pi)$ be a flow with discrete acting group $T$ and $\left(\tau(X), T, \pi_{*}\right)$ be the induced flow with the same acting group $T$. Then, between the projection maps $p r_{\pi(x, t)}=p r_{x t}$ and $p r_{x}$, the equation

$$
p r_{\pi(x, t)}\left(\pi_{*}(\zeta, t)\right)=t_{*}(\zeta)_{x t}=t_{*}\left(\zeta_{x}\right)=\pi_{*}\left(\zeta_{x}, t\right)
$$

holds.

A fixed point of the flow $(X, T, \pi)$ is a point $x_{0}$ such that $x_{0} t=x_{0}$, for all $t \in T$. By the definition of $\pi_{*}: \tau(X) \times T \rightarrow \tau(X)$, we can have $\pi_{*}(\mathcal{P}(X), t)=t_{*}(\mathcal{P}(X))=\mathcal{P}(X)$, and $\pi_{*}(\{\emptyset, X\}, t)=\{\emptyset, X\}$ for every $t \in T$. 
Theorem 3.3. Let $\left(\tau(X), T, \pi_{*}\right)$ be the induced flow with the same acting group $T$ by $(X, T, \pi)$. Then the phase space topology $\tau$ on $X$ is a fixed point of $\left(\tau(X), T, \pi_{*}\right)$.

Proof. Since $\pi^{t}=t:(X, \tau) \rightarrow(X, \tau)$ is a homeomorphism for every $t \in T, \pi_{*}(\tau, t)=t_{*}(\tau)=\left\{U \subset X \mid t^{-1}(U) \in \tau\right\}=\tau$. This completes the proof.

In any mathematical system, one is interested in the maps which respect the structure of the system. The appropriate maps in topological dynamics are those which are continuous and equivariant. To be precise, let $(X, T)$ and $(Y, T)$ be flows with same acting group. A homomorphism from $X$ to $Y$ is a continuous map $\phi: X \longrightarrow Y$ such that

$$
\phi(x t)=\phi(x) t \quad(x \in X, \quad t \in T) .
$$

Hence from the above theorems in section 2 and definitions, we have

TheOREM 3.4. Let $(X, T, \pi)$ be a flow with discrete acting group $T$ and $\left(\tau(X), T, \pi_{*}\right)$ be the induced flow with the same acting group $T$. Then the topology field $\zeta:(X, \tau) \longrightarrow\left(\tau(X), I n_{\tau}\right)$ is a homomorphism.

Proof. Since $t$ is a bijective, we can easily have

$$
\begin{aligned}
\zeta(x t) & =\{U \mid U \in \zeta, x t \in U\} \cup\{\emptyset\} \\
& =\left\{U \mid U \in \zeta, x \in t^{-1}(U)\right\} \cup\{\emptyset\} \\
& =\left\{U \mid x \in t^{-1}(U) \in \zeta\right\} \cup\{\emptyset\} \\
& =\left\{U \mid t^{-1}(U) \in \zeta_{x}\right\} \\
& =t_{*}\left(\zeta_{x}\right)=t_{*}(\zeta(x))=\pi_{*}(\zeta(x), t)=\zeta(x) t .
\end{aligned}
$$

The point $x$ and $y$ of a flow $X$ are said to be proximal [2] if there exists a net $\left\{t_{i}\right\}$ of elements of $T$ such that $\lim x t_{i}=\lim y t_{i}$. The points $x$ and $y$ are distal if either $x=y$ or $x$ and $y$ are not proximal. A homomorphism $\phi: X \rightarrow Y$ is said to be distal(proximal) provided that whenever $\phi\left(x_{1}\right)=$ $\phi\left(x_{2}\right)$, with $x_{1} \neq x_{2}$, then $x_{1}$ and $x_{2}$ are distal(proximal) [2].

Theorem 3.5. If $x$ and $y$ are proximal, then $\eta(x)$ and $\eta(y)$ are also proximal. Hence every topology field preserves the proximity. Similarly we can have every co topology field preserves the proximity.

Proof. We will prove theorem for case of the topology field. The other case follows with the same method. Let $G \in \tau$ be any open. Let $\lim \pi_{*}\left(\eta(x), t_{i}\right)=\lim t_{i_{*}}\left(\eta_{x}\right)=\lim \left\{U \in \tau \mid t_{i}^{-1}(U) \in \eta_{x}\right\} \in i(G)$, where $\left\{t_{i}\right\}$ is a net of elements of $T$. Then for almost all $i,\left\{U \in \tau \mid t_{i}^{-1}(U) \in\right.$ 
$\left.\eta_{x}\right\} \in i(G)$. Hence for almost all $i, G \in\left\{U \in \tau \mid t_{i}^{-1}(U) \in \eta_{x}\right\}$. That is, for almost all $i, t_{i}^{-1}(G) \in \eta_{x}$. Consequently $x \in t_{i}^{-1}(G), t_{i}(x) \in G$ for almost all $i$. Since $x$ and $y$ are proximal, $t_{i}(y) \in G$ for almost all $i$. This implies $y \in t_{i}^{-1}(G)$ for almost all $i$. Hence $\lim \pi_{*}\left(\eta(y), t_{i}\right) \in i(G)$.

By virtue of equation in the theorem 3.2 we have the following corollary.

Corollary 3.6. If $\eta$ and $\zeta$ are proximal, then $\eta(x)$ and $\zeta(x)$ are also proximal. Hence every projection preserves the proximity. Similarly we can have every co projection preserves the proximity.

Given a flow $X$, we can look at the family of closed and non-empty subsets of $X$. Each of these is called a subflow. We say that a flow is minimal if it has no proper subflow.

Theorem 3.7. Let $(X, T)$ be a minimal flow. Let $\eta: X \rightarrow \tau(X)$ be a topology field. Then the induced flow $\left(\tau(X), T, \pi_{*}\right)$ has minimal sets $\overline{\eta_{x_{0}} T}$. Moreover if $\eta_{x_{0}}=\zeta_{x_{0}}$, the minimal sets $\overline{\eta\left(x_{0}\right) T}$ and $\overline{\zeta\left(x_{0}\right) T}$ are equal.

Proof. Let $\eta \in \tau(X)$ be a topology field. Then $\overline{\eta_{x_{0}} T}$ is closed and non-vacuous. Moreover since $t_{*}$ is a homeomorphism, $\pi_{*}\left(\overline{\eta_{x_{0}} T}, t\right)=$ $t_{*}\left(\overline{\eta_{x_{0}} T}\right)=\overline{t_{*}\left(\eta_{x_{0}} T\right)}=\overline{\left(\eta_{x_{0}} T\right) t}=\overline{\eta_{x_{0}}(T t)}=\overline{\eta_{x_{0}} T}$. And we have $\overline{\eta_{x_{0}} T} \subset$ $\overline{\eta T}$. In fact, if $\zeta \in \overline{\eta_{x_{0}} T}$, for any subbasic nbd $i(G)$ of $\zeta$, there exists $t \in T$ such that $\pi_{*}\left(\eta_{x_{0}}, t\right)=t_{*}\left(\eta_{x_{0}}\right) \in i(G)$. Thus $t^{-1}(G) \in \eta_{x_{0}}$. Consequently $t^{-1}(G) \in \eta$. Hence $t_{*}(\eta) \in i(G)$. This implies $\zeta \in \overline{\eta T}$. Hence $\overline{\eta_{x_{0}} T}$ is minimal. Moreover if $\eta_{x_{0}}=\zeta_{x_{0}}$, then $\overline{\eta_{x_{0}} T}=\overline{\zeta_{x_{0}} T}$.

Theorem 3.8. Let $(X, \tau)$ be a distal minimal flow. And Assume that $\left(\tau(X)\right.$, Out $\left._{\tau}\right)$ is distal. If $\eta: X \rightarrow \tau(X)$ is a $T_{0}$ topology field, then it is a distal and proper homomorphism.

Proof. First by Theorem 3.4, we see that $\eta$ is a homomorphism. Let $x_{1}, x_{2} \in X$, with $x_{1} \neq x_{2}$ such that $\eta_{x_{1}}=\eta_{x_{2}}$. Since $\eta$ is $T_{0}$, there are $G_{1} \in \eta$ such that $x_{1} \in G_{1}, x_{2} \notin G_{1}$. This implies $G_{1} \in \eta_{x_{1}}, G_{1} \notin \eta_{x_{2}}$. This contradict to $\eta_{x_{1}}=\eta_{x_{2}}$. Hence there are no pairs of $x_{1}, x_{2} \in X$, with $x_{1} \neq x_{2}$ such that $\eta_{x_{1}}=\eta_{x_{2}}$. This means that every $T_{0}$ topology field $\eta$ is distal. And since $\eta$ is not onto, by virtue of Corollary in [1], we see that the topology field $\eta: X \rightarrow \tau(X)$ is proper. 


\section{Topology fields and topological organism}

Let $X$ be state space, $U$ the input space and $Y$ the output space. A deterministic dynamic system [8] is a complex

$$
\mathcal{S}=\{X, U, Y, \delta, \gamma\}
$$

where $\delta: X \times U \rightarrow X$ is the dynamics $\quad x_{t+1}=\delta\left(x_{t}, u_{t}\right)$ and $\gamma: X \rightarrow Y$ is the output map. Here we will give a generalization of this system whose output maps are topology fields.

Definition 4.1. Let $I$ be index set and $X_{t}$ state space at $t \in I$. Let $U$ be an input space. Let $X=\coprod_{t} X_{t}=\left\{\coprod x_{t} \mid x_{t} \in X_{t}\right\}$ be the total space, disjoint sum of spaces $X_{t}$. Denote $\tau(X)=\coprod \tau\left(X_{t}\right)=\left\{\tau_{t} \mid \tau \in \tau\left(X_{t}\right)\right\}$. The total topology $\tau$ on the total space $X$ is induced by $\tau(X)$

$$
\tau=\left\{G\left|G=\coprod G_{t}\right| G_{t} \in \tau_{t}\right\} .
$$

Hence we can get inner topology $I n_{\tau}$ on $\tau(X)$ induced by $\tau$ like as in section 2. A topological organism(or topological organic system) is a complex

$$
\mathcal{O} g=\{X, U, \tau(X), \delta, \gamma\}
$$

where $\delta: X \times U \rightarrow X$ is a dynamics and $\gamma$ is a topology field ("will be called output map") from $(X, \tau)$ to $\left(\tau(X) \cdot I n_{\tau}\right)$.

Here $x_{t} \in\left(X_{t}, \tau_{t}\right)$ is the local states in the topological states $\left(X_{t}, \tau_{t}\right)$ at time $t$. In this organic system the current state and input determine the next state and output.

This definition is a generalization of the definition of system [8], considering not only the states in the topological spaces, but also the outputs as beings topologies. Hence we have called this system topological organism(or topological organic system).

EXAMPLE 4.2. Let $I=R_{0}^{+}$be the set of nonnegative real numbers. Set $X_{t}=\left\{(x, y) \in R^{2} \mid x^{2}+y^{2}=t^{2}, y \geq 0\right\}$ be the subspace of Euclidean plane $R^{2}, t \in R_{0}^{+}$. Let $X=\coprod_{t} X_{t}$ and $\tau(X)=\coprod \tau\left(X_{t}\right)$. Then the total space may be represented by the sum of all half circles and point $(0,0)$ in the upper plane $\left\{(x, y) \in R^{2} \mid y \geq 0\right\}$. We give the total topology as above. Then the open set on total space can be represented by $G \cap X$ $=\coprod_{t}\left(G \cap X_{t}\right)$ for some open $G$ in $R^{2}$.

Define $\delta: X \times R_{0}^{+} \rightarrow X$ by $\delta\left(\left(x, \sqrt{t^{2}-x^{2}}\right), s\right)=\left(x, \sqrt{(t+s)^{2}-x^{2}}\right)$. It slides point $\left(x, \sqrt{t^{2}-x^{2}}\right) \in X_{t}$ to the point $\left(x, \sqrt{(t+s)^{2}-(x)^{2}}\right) \in X_{t+s}$ vertically. Hence if $s=1$, it slide the horizonal line $\{(x, 0) \mid x \in R\}$ to 
the curve $\{(x, \sqrt{2 x+1}) \mid x \in R\}$ vertically. Moreover for a fixed state $(0,0) \in X_{0}, \delta((0,0), s)=\{(0, s)\}$. Hence the image $\delta\left((0,0), R_{0}^{+}\right)$is the vertical half line $\{(0, s) \mid s \geq 0\}$. Finally if we define a natural topology field $\gamma:(X, \tau) \rightarrow\left(\tau(X)\right.$, Out $\left._{\tau}\right)$ by $\gamma\left(x_{t}\right)=\tau_{x_{t}}$, the we can get topological organism

$$
\mathcal{O} g=\left\{X, R_{0}^{+}, \tau(X), \delta, \gamma\right\} .
$$

In this example, the input space $R_{0}^{+}$is not a group and hence the dynamics is not a flow.

Considering a fixed input $s$, we can build maps

$$
\begin{gathered}
\delta_{s}: X \rightarrow X, \delta_{s}\left(x_{t}\right)=\delta\left(x_{t}, s\right), \\
\delta_{s *}^{\tau}: \tau(X) \rightarrow \tau(X), \delta_{s *}^{\tau}\left(\tau_{t}\right)=\left\{G \in \tau \mid \delta_{s}{ }^{-1}(G) \in \tau_{t}\right\}
\end{gathered}
$$

and will be called input map induced by input $s$ and induced map by input map, respectively.

Similarly consider a fixed state $x_{t} \in\left(X_{t}, \tau_{t}\right)$, we can build maps

$$
\begin{aligned}
& \bar{\delta}_{t}: U \rightarrow X, \quad \bar{\delta}_{t}(s)=\delta\left(x_{t}, s\right), \\
& \bar{\gamma}_{t}: U \rightarrow \tau(X), \bar{\gamma}_{t}(s)=\gamma_{x_{t+s}}
\end{aligned}
$$

and will be called input map and output map induced by state $x_{t}$ respectively.

Therefore, $\bar{\delta}_{t}(s)$ and $\bar{\gamma}_{t}(s)$ are computed by starting the system in state $x_{t} \in\left(X_{t}, \tau_{t}\right)$, feeding in the input sequence $s$ and looking at the final state.

Definition 4.3. The topological organism $\mathcal{O}_{g}$ is called totally reachable from the state $x_{t} \in\left(X_{t}, \tau_{t}\right)$ if the map $\bar{\delta}_{t}$ is onto, that is $\delta\left(x_{t}, U\right)$ $=X$. And the topological organism $\mathcal{O}_{g}$ is called almost reachable from the state $x_{t} \in\left(X_{t}, \tau_{t}\right)$ if $\overline{\delta\left(x_{t}, U\right)}=X$. And topological organism $\mathcal{O}_{g}$ is called globally reachable from the state $\left(X_{t}, \tau_{t}\right)$ if $\delta\left(X_{t}, U\right)=X$ and almost globally reachable from the state $\left(X_{t}, \tau_{t}\right)$ if $\overline{\delta\left(X_{t}, U\right)}=X$. Hence (almost) totally reachable organism is (almost) globally reachable.

Above concept of almost reachable is closely related to the concepts of almost periodic and minimal in the topological dynamics and is central concept of the topological dynamics. Let $x$ be an almost periodic point of $(X, R)$ where $X$ is the plane and $R$ is reals. Then the Poincare-Bendixon theorem state $\overline{x R}$ is either a point or a circle.

Definition 4.4. The topological organism $\mathcal{O}_{g}$ is called observable if the map $\bar{\gamma}_{t}$ is one to one. 
The topological organism defined in Example 1 is observable and not totally reachable from the one any state $x_{t} \in\left(X_{t}, \tau_{t}\right)$. Moreover it is not globally reachable from the any state $\left(X_{t}, \tau_{t}\right)$.

The next example tells us there exist almost globally reachable from the any state $\left(X_{t}, \tau_{t}\right)$.

EXAmple 4.5. Let $R$ be reals and $X_{t}=\left\{\left(e^{2 \pi i t}, e^{2 \pi i u}\right) \mid u \in R\right\}$ for $t \in R$. Let $X=\coprod_{t} X_{t}$ and $\tau(X)=\coprod \tau\left(X_{t}\right)$. Then the total space may be representant by torus $S^{1} \times S^{1}$ in the Euclidean space $R^{3}$. We give the total topology as above. Then the open set on total space can be represented by $G \cap X=\coprod_{t}\left(G \cap X_{t}\right)$ for some open $G$ in $R^{3}$. Consider rationals $Q$ as input space. Define $\delta: X \times Q \rightarrow X$ by $\delta\left(\left(e^{2 \pi t}, e^{2 \pi i u}\right), s\right)=$ $\left(e^{2 \pi(t+s)}, e^{2 \pi i u}\right)$. It slides point $\left.\left(e^{2 \pi i t}, e^{2 \pi i u}\right)\right) \in X_{t}$ to the point $\left(e^{2 \pi(t+s)}\right.$, $\left.e^{2 \pi i u}\right) \in X_{t+s}$ along the circle in the torus. Hence if $s=1$, it slide the circle $X_{t}$ to the circle $X_{t+s}$ along the horizontal circle. Moreover for a fixed state $(0,0) \in X_{0}, \delta((0,0), s)=\left\{\left(e^{2 \pi s}, 0\right)\right\}$. Hence the image $\delta((0,0), Q)$ is the dense subset $\left\{\left(e^{2 \pi i s}, 0\right) \mid s \in Q\right\}$ of the horizontal circle $\left\{\left(e^{2 \pi i r}, 0\right) \mid r \in R\right\}$. Moreover $\overline{\delta\left(\left(X_{t}, \tau\right), Q\right)}=X$. Finally we can get a natural topology field $\gamma:(X, \tau) \rightarrow\left(\tau(X), \operatorname{In}_{\tau}\right)$ by $\gamma\left(e^{2 \pi t}, e^{2 \pi i u}\right)=$ $\tau_{\left(e^{2 \pi t}, e^{2 \pi i u}\right)}$. Consequently we can have a topological organism

$$
\mathcal{O} g=\{X, Q, \tau(X), \delta, \gamma\} .
$$

Thus this organism is almost globally reachable.

In this example, the input space $Q$ is a additive group and hence the dynamics is a flow. We will give a totally reachable and globally reachable example.

EXAmple 4.6. For the same index space and state spaces in the above Example, consider another input space $R \times R$. Define $\delta: X \times R \times$ $R \rightarrow X$ by $\delta\left(\left(e^{2 \pi t}, e^{2 \pi i u}\right),(s, w)\right)=\left(e^{2 \pi(t+s)}, e^{2 \pi i(u+w)}\right)$. It changes any local state $\left(e^{2 \pi i t}, e^{2 \pi i u}\right) \in X_{t}$ to the local state $\left(e^{2 \pi i(t+s)}, e^{2 \pi i(u+w)}\right)$ in $X_{t+s}$ along the circular helix in the torus. Hence if $s=1, w=1$, it changes the local state $\left(e^{2 \pi i t}, e^{2 \pi i u}\right) \in X_{t}$ to the local state $\left(e^{2 \pi(t+1)}\right.$, $\left.e^{2 \pi i(u+1)}\right)$ on the circle $X_{t+s}$ along the circular helix. Moreover for a fixed local state $(0,0) \in X_{0}, \delta((0,0),(s, w))=\left(e^{2 \pi i s}, e^{2 \pi i w}\right)$. Hence the image $\delta((0,0), R \times R)$ is the space $S^{1} \times S^{1}$. And if $s=1, w=0$, it changes the local state $\left.\left(e^{2 \pi i t}, e^{2 \pi i u}\right)\right) \in X_{t}$ to the local state $\left(e^{2 \pi(t+1)}, e^{2 \pi i u}\right)$ on the circle $X_{t+1}$ along the circle. Moreover for a fixed local state $(1,1) \in X_{0}$, $\delta((1,1),(s, 0))=\left(e^{2 \pi i s}, 1\right)$. Hence the image $\delta((1,1), R \times\{0\})$ is the circle $S^{1} \times\{1\}$. Finally we get a topology field $\gamma:(X, \tau) \rightarrow\left(\tau(X)\right.$, Out $\left._{\tau}\right)$ by 
$\gamma\left(e^{2 \pi t}, e^{2 \pi i u}\right)=\tau_{\left(e^{2 \pi t}, e^{2 \pi i u}\right)}$. Thus we can get topological organism

$$
\mathcal{O} g=\{X, R \times R, \tau(X), \delta, \gamma\} .
$$

Hence this topological organism is totally reachable and globally reachable example.

\section{References}

[1] J. Auslander, Homomorphisms Of Minimal Transformation Groups, Topology 9 (1970), 195-203

[2] J. Auslander, Minimal Flows and Their Extensions, North-Holland Mathematics Studies 153, 1988

[3] R. Ellis, Lectures on Topological Dynamics, W. A. Benjamin, Inc., 1969.

[4] D. H. Gottlieb, Lectures on Vector Fields and the Unity of Mathematics, Preprint.

[5] G. Grätzer, General Lattice Theory, Basel: Birkhäuser, 1978.

[6] J. Hartmanis, On the lattice of topologies, Canad. J. Math. 10 (1958), 547-553.

[7] J. R. Kim and M. H. Woo, Topology Fields and Fixed Points of Flows, Research and Exposition in Mathematics 27 (2003), 73-81.

[8] C. V. Negoita and D. A. Ralescu, Applications of Fuzzy Sets to Systems Analysis, A Halsted Press Book, 1975

[9] P. T. Johnstone, Stone spaces, Cambridge University Press, 1982.

[10] R. E. Larson and S. J. Andima, The lattice of topologies: A survey, Rockey mountain Jour. Math. 5 (1975), no. 2, Spring.

[11] A. K. Steiner, The lattice of topologies: Structure and Complementation, Trans. Amer. Math. Soc. 3 (1963), 167-171.

Department of Mathematics

Kookmin University

Seoul 136-702, Republic of Korea

E-mail: kimjr@kookmin.ac.kr 